\title{
Efficacy of sorafenib in advanced differentiated and medullary thyroid cancer: experience in a Turkish population
}

\author{
Mustafa Benekli' \\ Suayib Yalcin ${ }^{2}$ \\ Metin Ozkan ${ }^{3}$ \\ Emin Tamer Elkiran ${ }^{4}$ \\ Alper Sevinc ${ }^{5}$ \\ Devrim Cabuk ${ }^{6}$ \\ Hasan Senol Coskun ${ }^{7}$ \\ Berna Oksuzoglu ${ }^{8}$ \\ Banu Bayar? \\ Akif Akbulat ${ }^{9}$ \\ Ahmet Ozet' \\ On behalf of Turkish Thyroid \\ Cancer Study Group \\ 'Department of Medical Oncology, Gazi \\ University Faculty of Medicine, Ankara, \\ ${ }^{2}$ Department of Medical Oncology, \\ Hacettepe University Faculty of Medicine, \\ Ankara, ${ }^{3}$ Department of Medical Oncology, \\ Erciyes University Faculty of Medicine, \\ Kayseri, ${ }^{4}$ Department of Medical Oncology, \\ Inonu University Faculty of Medicine, \\ Malatya, ${ }^{5}$ Department of Medical Oncology, \\ Gaziantep University Faculty of Medicine, \\ Gaziantep, ${ }^{6}$ Department of Medical \\ Oncology, Kocaeli University Faculty of \\ Medicine, Kocaeli, ${ }^{7}$ Department of Medical \\ Oncology, Akdeniz University Faculty of \\ Medicine, Antalya, ${ }^{8}$ Department of Medical \\ Oncology, Ankara Oncology Training and \\ Research Hospital, Ankara, ${ }^{9}$ Ministry of \\ Health of Turkey, General Directorate \\ of Pharmaceuticals and Medical Devices, \\ Ankara, Turkey
}

Correspondence: Mustafa Benekli

Department of Medical Oncology,

Gazi University Faculty of Medicine,

Beșevler, Ankara, Turkey

Tel +90312 2025825

$\mathrm{Fax}+903122158710$

Email mbenekli@gmail.com
This article was published in the following Dove Press journal:

OncoTargets and Therapy

I5 December 2014

Number of times this article has been viewed

Background: Antivascular endothelial growth factor tyrosine kinase inhibitors have been used recently in the treatment of advanced differentiated thyroid cancer (DTC) and medullary thyroid cancer (MTC). Off-label sorafenib is used in Turkey with special permission by the Ministry of Health for this indication.

Patients and methods: Patients with advanced DTC and MTC were retrospectively identified from the Turkish Ministry of Health database. Data on these patients were prospectively collected before permission is granted to use sorafenib.

Results: Thirty patients with complete data were analyzed: 14 DTC (papillary number $[\mathrm{n}]=10$; follicular $\mathrm{n}=4$ ) and 16 MTC. The median age of the patients was 57 years (range: 28-79 years), and there were 18 males and 12 females. All DTC patients were iodine refractory and had received a median three doses of radioactive iodine (range: 1-7 doses). Sorafenib was used for a median of 12 months (range: 1-49 months). The overall response rate was $20 \%$, all partial responses, with no complete response. The overall response rate was $14 \%$ in DTC and $25 \%$ in MTC patients. The median progression-free survival (PFS) was 17.1 months $(95 \%$ confidence interval $[\mathrm{CI}]$ : 7.3-26.8) and overall survival (OS) was not reached. The 2-year PFS and OS were 39\% and 68\%, respectively. DTC and MTC patients had similar survival outcomes: median PFS of 21.3 months (95\% CI: 5.8-36.7) versus 14.5 months (95\% CI: 3.7-25.2), respectively $(P=0.36)$, with the median OS not reached in either group $(P=0.17)$. Tumor marker levels did not have any prognostic or predictive role. The toxicity profile was similar to that of other sorafenib trials.

Conclusion: Sorafenib is an effective and well-tolerated treatment in advanced thyroid cancers.

Keywords: advanced thyroid cancer, sorafenib, overall survival

\section{Introduction}

Thyroid cancer is the most common endocrine cancer; it is the ninth most common cancer in the United States and the seventh most common cancer in Turkey. ${ }^{1,2}$ The main histopathologic types are differentiated thyroid cancer (DTC) (including papillary, follicular, and Hurtle), medullary thyroid cancer (MTC), and anaplastic thyroid cancer. Advanced thyroid cancer is a lethal disease with no effective systemic therapy. Novel therapies are needed for radioiodine (RAI)-refractory DTC and advanced MTC.

Thyroid cancers are vascular tumors that overexpress vascular endothelial growth factor (VEGF). ${ }^{3,4}$ The definition of molecular pathogenesis and the role of downstream mitogenic signal transduction pathways, including mitogen-activated protein kinase in thyroid cancers, has led to the introduction of multikinase inhibitors that block tyrosine kinases in the treatment of metastatic DTC and MTC. ${ }^{5}$ In this context, several tyrosine kinase inhibitors (TKIs) have been used with various results. ${ }^{6-19}$ 
Sorafenib is an orally active multikinase inhibitor of the tyrosine kinases including BRAF, rearranged during transfection (RET), FLT-3, KIT, VEGFR-1, VEGFR-2, VEGFR-3, and platelet-derived growth factor receptor- $\beta{ }^{20}{ }^{20}$ Sorafenib is one of the most commonly utilized agents for this indication. ${ }^{10-19}$ Several Phase II studies ${ }^{10-15}$ and retrospective observations ${ }^{16-18}$ proved its efficacy and have led to the design of the Phase III DECISION trial. ${ }^{19}$ The DECISION trial showed that sorafenib is a viable therapeutic option in advanced thyroid cancers. Sorafenib has been used in Turkey with special permission from the Ministry of Health for this off-label indication in the treatment of these patients. We report herein the Turkish experience with off-label sorafenib in patients with metastatic DTC and MTC.

\section{Patients and methods Study design}

Patients with advanced DTC and MTC were retrospectively identified from the Turkish Ministry of Health database. Off-label uses of all medications are only possible with special permission from the Turkish Ministry of Health. Data on these patients were prospectively collected before permission is granted to use the drugs. Therefore, it was possible to reach all of the patients using a specific drug for a specific indication from the database. Sorafenib has been used in the treatment of metastatic thyroid cancer in this context, and basic clinical information about the patients could be obtained retrospectively through this system. Ethics committee approval was obtained prior to the study.

Advanced thyroid cancer patients had the following inclusion criteria: iodine-refractory disease in differentiated cancers; measurable disease by the Response Evaluation Criteria in Solid Tumors (RECIST); and an Eastern Cooperative Oncology Group (ECOG) performance status of $0-1$. Patients who did not meet these criteria were not evaluated and were excluded from the analysis.

Sorafenib was given per the discretion of attending physicians. The usual starting dose of $400 \mathrm{mg}$ twice daily (bid) was most commonly applied. Clinical information, including efficacy and toxicity data, were obtained retrospectively from individual medical records. Toxicity was graded according to Common Terminology Criteria for Adverse Events version 3.0 (CTCAE v3.0).

\section{Statistics}

The primary objective was to assess the overall response rate (ORR) using RECIST. Additional endpoints were progression-free survival (PFS), overall survival (OS), and adverse events (AEs). PFS was assessed as the time the study drug was started until documented progression or death, whichever came first. OS was defined as the period from the first day of treatment until the date of the last follow-up or death. The probabilities of PFS and OS were estimated using the Kaplan-Meier method and were compared by log-rank test. $P<0.05$ was considered statistically significant.

\section{Results}

\section{Patients}

A total of 42 Caucasian patients were obtained from the database. Twelve of them were not included in the analysis because of the refusal by the treating physicians to participate in the study (number $[n]=5)$, missing medical records $(n=5)$, or the patients not using sorafenib after approval $(n=2)$.

Thirty patients were appropriately analyzed. Demographics and baseline characteristics of these patients are given in Table 1. Of these, 14 had DTC (papillary, $n=10$; follicular, $\mathrm{n}=4$ ) and 16 had MTC (one patient tested positive for the RET proto-oncogene). The median age of the patients was

Table I Patient characteristics

\begin{tabular}{|c|c|c|c|}
\hline Characteristics & $\begin{array}{l}\text { Patients } \\
(n=30)(\%)\end{array}$ & $\begin{array}{l}\text { DTC } \\
(n=\mid 4)(\%)\end{array}$ & $\begin{array}{l}\text { MTC } \\
(n=16)(\%)\end{array}$ \\
\hline $\begin{array}{l}\text { Median age, } \\
\text { years (range) }\end{array}$ & 57 (28-79) & $58.5(43-79)$ & $48.5(28-72)$ \\
\hline $\operatorname{Sex}(M / F)$ & $18 / 12(60 / 40)$ & $7 / 7(50 / 50)$ & II/5 (68.7/3I.2) \\
\hline \multicolumn{4}{|c|}{ Baseline ECOG performance status } \\
\hline Unknown & $4(13.3)$ & $2(14.2)$ & $2(12.5)$ \\
\hline 0 & $15(50)$ & $6(42.8)$ & $9(56.2)$ \\
\hline I & $10(33.3)$ & $5(35.7)$ & $5(3 \mid .2)$ \\
\hline 2 & I (3.3) & I (7.I) & \\
\hline \multicolumn{4}{|l|}{ Tumor histology } \\
\hline Differentiated (total) & I 4 (46.6) & & \\
\hline Papillary & $10(71.4)$ & & \\
\hline Follicular & $4(28.6)$ & & \\
\hline Medullary & $16(53.3)$ & & \\
\hline \multicolumn{4}{|l|}{ Metastatic sites } \\
\hline Lung & $16(53.3)$ & $8(57.1)$ & $8(50)$ \\
\hline Bone & II (36.6) & $6(42.8)$ & $5(3 \mid .2)$ \\
\hline Lymph nodes & $10(33.3)$ & $5(35.7)$ & $5(31.2)$ \\
\hline Liver & $6(20)$ & & $6(37.5)$ \\
\hline Other & $2(6.6)$ & I (7.I) & I (6.2) \\
\hline \multicolumn{4}{|c|}{ Number of metastatic sites } \\
\hline I & 14 (46.6) & $6(42.8)$ & $8(50)$ \\
\hline 2 & $12(40)$ & $7(50)$ & $5(3 \mid .2)$ \\
\hline$\geq 3$ & $4(13.3)$ & I (7.I) & $3(18.7)$ \\
\hline \multicolumn{4}{|l|}{ Previous therapies } \\
\hline Surgery & $23(77)$ & $13(92.8)$ & $10(62.5)$ \\
\hline Radiotherapy & $21(70)$ & $8(57.1)$ & $13(81.2)$ \\
\hline Radioiodine therapy & 14 & $100(100)$ & \\
\hline Chemotherapy & $24(80)$ & $9(64.2)$ & 15 (93.7) \\
\hline
\end{tabular}

Abbreviations: $n$, number; DTC, differentiated thyroid cancer; MTC, medullary thyroid cancer; ECOG, Eastern Cooperative Oncology Group; M, male; F, female. 
57 years (range: 28-79 years). Eighteen patients were males $(60 \%)$ and 12 were females (40\%). All DTC patients were iodine refractory and had received a median of three doses of RAI (range: 1-7). Twenty-three patients (77\%) had had surgery and most patients $(70 \%)$ had received radiotherapy to the neck (33\%) and metastatic sites for palliation. Most of the patients had received chemotherapy $(\mathrm{n}=24 ; 80 \%)$ - most commonly, cisplatin and doxorubicin/ epirubicin alone or in combination with cisplatin $(n=19$; $79 \%)$. Only six patients (20\%) had received two or more lines of chemotherapy.

Sorafenib was started at a $400 \mathrm{mg}$ bid dose in 24 patients (80\%). The median sorafenib dose was $800 \mathrm{mg}$ per day (range: 400-800 doses). Seven patients required a dose reduction for AEs, and the dose was increased to $400 \mathrm{mg}$ bid in one patient. Sorafenib was used for a median of 12 months (range: 1-49 months). The median duration of sorafenib therapy in DTC and MTC patients was 15 months (range: 2-49 months) and 9 months (range: 1-27 months), respectively.

\section{Efficacy}

In the entire study population, the ORR was $20 \%$; all were partial responses with no complete response. An additional ten patients $(33 \%)$ had stable disease (SD) with a clinical benefit rate (clinical benefit rate $=$ ORR + SD) of $53 \%$. The ORR was $14 \%$ in DTC and $25 \%$ in MTC patients. SD was observed in six DTC patients and four MTC patients.

In the survival analysis, the median PFS was 17.1 months (95\% confidence interval [CI]: 7.3-26.8) and the OS was not reached. The 2-year PFS and OS were 39\% and 68\%, respectively. The median PFS in the DTC and MTC groups was similar (21.3 months [95\% CI: 5.8-36.7] versus 14.5 months [95\% CI: 3.7-25.2], respectively; $P=0.36$ ) (Figure 1). The median OS was not reached in either group $(P=0.17)$ (Figure 2).

Calcitonin and thyroglobulin levels were obtained in some patients at both baseline and at one time point after sorafenib administration. Of 14 DTC patients, eight had thyroglobulin measurements and only three of them had a decrease in thyroglobulin levels. These three patients had SD as a tumor response. Similarly, of the 16 MTC patients, 12 had calcitonin measurements. Nine of these had an increase or no change in calcitonin levels, whereas only three patients had a decrease. Of these three patients, one had a partial response, one had SD and one had PD. There was no correlation of tumor marker levels with sorafenib efficacy.

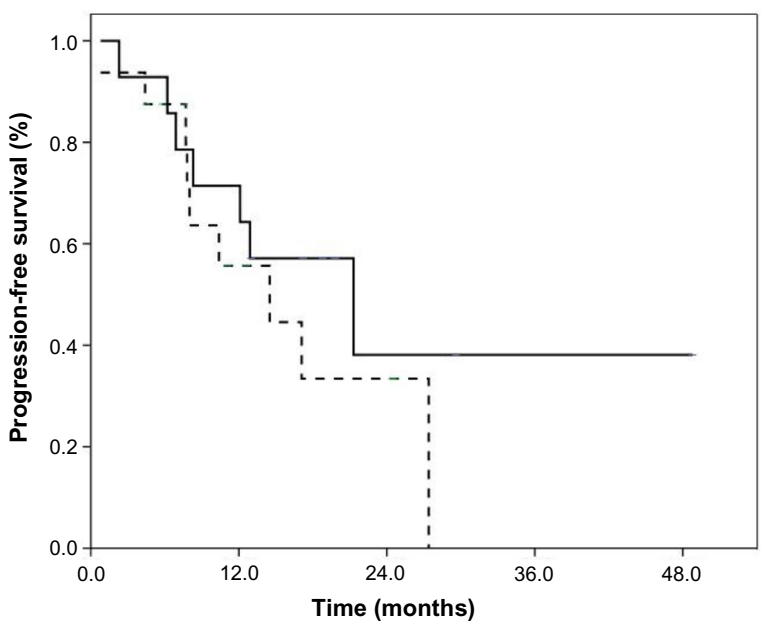

Figure I Kaplan-Meier analysis of progression-free survival in DTC and MTC patients (solid line DTC, dotted line MTC patients).

Note: No statistically significant difference was found between groups with the log-rank test ( $P=\mathrm{NS})$.

Abbreviations: DTC, differentiated thyroid cancer; MTC, medullary thyroid cancer; NS, not significant.

\section{Adverse events}

The toxicity profile was similar to those of other sorafenib trials. Grade 1-2 AEs might have been overlooked because of the retrospective nature of the data collection. The most common grade 3-4 toxicities are summarized in Table 2. One patient discontinued therapy because of fatigue and handfoot syndrome. In addition, the sorafenib dose was reduced in seven patients (23\%) because of AEs.

\section{Discussion}

Treatment of advanced thyroid cancers is challenging once they are RAI refractory in DTC or unresectable with curative

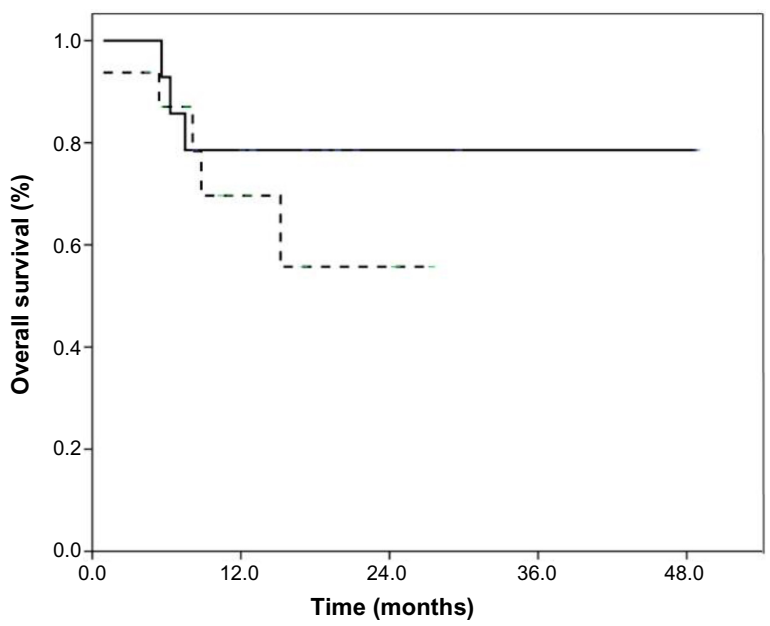

Figure 2 Kaplan-Meier analysis of overall survival in DTC and MTC patients (solid line DTC, dotted line MTC patients).

Note: Overall survival has not been reached in either group $(P=N S)$.

Abbreviations: DTC, differentiated thyroid cancer; MTC, medullary thyroid cancer; NS, not significant. 
Table 2 Adverse events grade 3 or 4

\begin{tabular}{ll}
\hline Characteristics & Patients $(\mathbf{n}=\mathbf{3 0})(\%)$ \\
\hline Hand-foot syndrome & $2(22)$ \\
Skin desquamation & $\mathrm{I}(\mathrm{II})$ \\
Fatigue & $2(22)$ \\
Diarrhea & $2(22)$ \\
Infection & $2(22)$ \\
Neuropathy & $\mathrm{I}(\mathrm{II})$ \\
Weight loss & $\mathrm{I}(\mathrm{II})$ \\
Nausea & $\mathrm{I}(\mathrm{II})$ \\
\hline
\end{tabular}

Abbreviation: $\mathrm{n}$, number.

intent in MTC. Therefore, novel treatment options are urgently needed. The recent demonstration of activity with small-molecule TKIs has led to vigorous investigations in this field with promising results. ${ }^{10-19}$

Sorafenib is the most promising of these TKIs. Demonstration of meaningful clinical activity in small Phase II trials has led to the design of the placebo-controlled Phase III DECISION trial in DTC. ${ }^{19}$ The trial enrolled 417 patients with advanced DTC refractory to RAI. The primary endpoint of the study was reached; the median PFS was increased by $41 \%$ (10.8 months versus 5.8 months) with sorafenib compared to placebo (hazard ratio $=0.587$; $P<0.0001)$. Unfortunately, there was no statistically significant OS benefit because the patients taking placebo were permitted to cross over to the open-label sorafenib treatment upon progression. It should be noted that the median OS has not yet been reached in either arm. Consequently, based on these results sorafenib was approved by the US Food and Drug Administration in November 2013 for the treatment of advanced DTC.

Our results of sorafenib activity in DTC patients were similar to the reports in the literature. The ORR of $14 \%$ in DTC patients was almost identical to that reported in the DECISION trial (12\%). ${ }^{19}$ Moreover, the median PFS of 17.1 months was in the range of those in published trials, which ranged from 9 months to not reached. Median OS has not yet been reached in our study, similar to these data.

Sorafenib was also tested in MTC patients in various studies with similar efficacy to DTC. ${ }^{14-16}$ Sorafenib use in MTC was reported in two Phase II studies and in one retrospective case series. ${ }^{14-16}$ Our results in MTC were in accordance with these published results, supporting the efficacy of sorafenib in these patients. Other TKIs that have been tested in this indication include vandetanib and cabozantinib, with available Phase III data and consequent US FDA approval. ${ }^{21,22}$ It seems that sorafenib is as effective as vandetanib or cabozantinib in this indication, despite the lack of direct comparisons. However, a lack of Phase III data in advanced MTC makes sorafenib an unattractive choice. Because the use of placebo is no longer an option, comparative Phase III trials of sorafenib with vandetanib or cabozantinib should be undertaken.

Tumor marker levels did not have any prognostic or predictive radiologic response significance in our study. This issue falls into a gray area because data are rather unclear with conflicting Phase II trial results. ${ }^{12,14}$ Having said that, our results further support the general opinion of not depending on biomarker level changes during treatment. Therefore, tumor markers, thyroglobulin and calcitonin, should not be taken into consideration in response evaluation and decisionmaking processes.

Sorafenib was reasonably well tolerated. Its toxicity profile was in accordance with that of previous sorafenib trials. Most of the patients presented with grade 1-2 AEs, mostly hand-foot syndrome, and these were easily managed with supportive care and dose reductions (23\%) where necessary. Grade 3 and 4 AEs were rare, necessitating drug discontinuation in one patient.

The present study has several drawbacks. The retrospective nature of our study limits its merit. We have to acknowledge that a lack of standardized frequency of response assessment in various centers might have influenced PFS and OS durations. Additionally, AEs might have been underestimated in terms of grade $1 / 2$ toxicities because of the physicians' lack of attention to recording mild toxicities in the medical records. Nevertheless, all grade 3/4 severe reactions were precisely recorded. Likewise, the survival outcomes achieved in our study are within the range of other published results in comparable patient populations. Our report reflects actual clinical experience and is free of innate trial problems, such as patient selection bias and closer follow-up, resulting in better clinical outcomes. Most importantly, this article includes real-life results of consecutive patients with advanced thyroid cancer who used off-label sorafenib in a given time period.

\section{Conclusion}

In summary, we demonstrated that sorafenib is an active agent with a tolerable toxicity profile in the treatment of patients with advanced DTC and MTC. These results showed that its efficacy in this indication is reproducible in real life. The equal efficacy of sorafenib both in DTC and MTC patients is an important observation that demands further exploration of its activity in advanced MTC. 


\section{Acknowledgments}

Turkish Thyroid Cancer Study Group Collaborators (in alphabetical order according to last name): Dilsen Colak (Diskapi Education and Research Hospital); Turkkan Evrensel (Uludag University); Guzin Gonullu (19 Mayis University); Saadettin Kilickap (Cumhuriyet University); Seref Komurcu (Bayindir Hospital); Fatih Kose (Baskent University Adana Hospital); Bulent Orhan (Acibadem Bursa Hospital); and Mustafa Ozguroglu (Cerrahpasa University).

\section{Disclosure}

The authors report no conflicts of interest in this work.

\section{References}

1. Siegel R, Naishadham D, Jemal A. Cancer statistics, 2013. CA Cancer J Clin. 2013;63(1):11-30.

2. Cancer Incidence in 2009. Available from: http://kanser.gov.tr/dairefaaliyetleri/kanser-istatistikleri.html. Accessed November 7, 2014.

3. Capp C, Wajner SM, Siqueira DR, Brasil BA, Meurer L, Maia AL. Increased expression of vascular endothelial growth factor and its receptors, VEGFR-1 and VEGFR-2, in medullary thyroid carcinoma. Thyroid. 2010;20(8):863-871.

4. Kilicarslan AB, Ogus M, Arici C, Pestereli HE, Cakir M, Karpuzoglu G. Clinical importance of vascular endothelial growth factor (VEGF) for papillary thyroid carcinomas. APMIS. 2003;111(3):439-443.

5. Gild ML, Bullock M, Robinson BG, Clifton-Bligh R. Multikinase inhibitors: a new option for the treatment of thyroid cancer. Nat Rev Endocrinol. 2011;7(10):617-624.

6. Sherman SI, Wirth LJ, Droz JP, et al; Motesanib Thyroid Cancer Study Group. Motesanib diphosphate in progressive differentiated thyroid cancer. $N$ Engl J Med. 2008;359(1):31-42.

7. Cohen EE, Rosen LS, Vokes EE, et al. Axitinib is an active treatment for all histologic subtypes of advanced thyroid cancer: results from a phase II study. J Clin Oncol. 2008;26(29):4708-4713.

8. Bible KC, Suman VJ, Molina JR, et al; Endocrine Malignancies Disease Oriented Group; Mayo Clinic Cancer Center; Mayo Phase 2 Consortium. Efficacy of pazopanib in progressive, radioiodine-refractory, metastatic differentiated thyroid cancers: results of a phase 2 consortium study. Lancet Oncol. 2010;11(10):962-972.
9. Wells SA Jr, Robinson BG, Gagel RF, et al. Vandetanib in patients with locally advanced or metastatic medullary thyroid cancer: a randomized, double-blind phase III trial. J Clin Oncol. 2012;30(2):134-141.

10. Gupta-Abramson V, Troxel AB, Nellore A, et al. Phase II trial of sorafenib in advanced thyroid cancer. J Clin Oncol. 2008;26(29):4714-4719.

11. Keefe SM, Troxel AB, Rhee S, et al. Phase II trial of sorafenib in patients with advanced thyroid cancer [abstract]. J Clin Oncol. 2011; 29 Suppl:abstract 5562.

12. Kloos RT, Ringel MD, Knopp MV, et al. Phase II trial of sorafenib in metastatic thyroid cancer. J Clin Oncol. 2009;27(10):1675-1684.

13. Schneider TC, Abdulrahman RM, Corssmit EP, Morreau H, Smit JW, Kapiteijn E. Long-term analysis of the efficacy and tolerability of sorafenib in advanced radio-iodine refractory differentiated thyroid carcinoma: final results of a phase II trial. Eur J Endocrinol. 2012; 167(5):643-650.

14. Lam ET, Ringel MD, Kloos RT, et al. Phase II clinical trial of sorafenib in metastatic medullary thyroid cancer. $J$ Clin Oncol. 2010;28(14):2323-2330.

15. Ahmed M, Barbachano Y, Riddell A, et al. Analysis of the efficacy and toxicity of sorafenib in thyroid cancer: a phase II study in a UK based population. Eur J Endocrinol. 2011;165(2):315-322.

16. Capdevila J, Iglesias L, Halperin I, et al. Sorafenib in metastatic thyroid cancer. Endocr Relat Cancer. 2012;19(2):209-216.

17. Cabanillas ME, Waguespack SG, Bronstein Y, et al. Treatment with tyrosine kinase inhibitors for patients with differentiated thyroid cancer: the MD Anderson experience. J Clin Endocrinol Metab. 2010;95(6):2588-2595.

18. Marotta V, Ramundo V, Camera L, et al. Sorafenib in advanced iodinerefractory differentiated thyroid cancer: efficacy, safety and exploratory analysis of role of serum thyroglobulin and FDG-PET. Clin Endocrinol (Oxf). 2013;78(5):760-767.

19. Brose MS, Nutting CM, Jarzab B, et al; DECISION investigators Sorafenib in radioactive iodine-refractory, locally advanced or metastatic differentiated thyroid cancer: a randomised, double-blind, phase 3 trial. Lancet. 2014;384(9940):319-328.

20. Nexavar (sorafenib) [prescribing information]. Bayer Pharmaceuticals. Available from: http://berlex.bayerhealthcare.com/html/products/pi/ Nexavar_PI.pdf.

21. Wells SA, Robinson BG, Gagel RF, et al. Vandetanib in patients with locally advanced or metastatic medullary thyroid cancer: a randomized, double-blind phase III trial. J Clin Oncol. 2012;30(2):134-141.

22. Elisei R, Schlumberger MJ, Müller SP, et al. Cabozantinib in progressive medullary thyroid cancer. J Clin Oncol. 2013;31(29):3639-3646.
OncoTargets and Therapy

\section{Publish your work in this journal}

OncoTargets and Therapy is an international, peer-reviewed, open access journal focusing on the pathological basis of all cancers, potential targets for therapy and treatment protocols employed to improve the management of cancer patients. The journal also focuses on the impact of management programs and new therapeutic agents and protocols on

\section{Dovepress}

patient perspectives such as quality of life, adherence and satisfaction The manuscript management system is completely online and includes a very quick and fair peer-review system, which is all easy to use. Visit http://www.dovepress.com/testimonials.php to read real quotes from published authors. 